\title{
STABILITY OF VISCOUS SCALAR SHOCK FRONTS IN SEVERAL DIMENSIONS
}

\author{
JONATHAN GOODMAN
}

\begin{abstract}
We prove nonlinear stability of planar shock front solutions for viscous scalar conservation laws in two or more space dimensions. The proof uses the "integrated equation" and an effective equation for the motion of the front itself. We derive energy estimates that balance terms from the integrated equation with terms from the front motion equation.
\end{abstract}

In this paper we prove that viscous shock profiles for scalar conservation laws are stable in two or more space dimensions. These multidimensional stability questions are separate from their one dimensional analogues because of the possibility of transverse instabilities such as those that occur in combustion fronts [Lu] and in shock waves with phase changes. The proof here is a rigorous version of arguments that are used to derive effective equations (such as the Kuramoto-Sivashinsky equation) to describe the behavior of fronts.

The one dimensional stability for scalar conservation laws was proven by $\mathrm{Il}^{\prime}$ in and Oleinik [IO] using the "integrated equation" (see below) and a maximum principle. Another proof, based on weighted norms and spectral theory for the linearized problem, was given by Sattinger [S]. The multidimensional stability proof below has more in common with the stability proofs for systems of conservation laws in one space dimension begun by Kawashima and Matsumura $[\mathrm{KM}]$ and Goodman [Go] and completed by Liu [Li]. These proofs use $L_{2}$ energy estimates for the integrated equation.

We consider equations of the form

$$
u_{t}+f(u)_{x}+g(u)_{y}=u_{x x}+u_{y y},
$$

where $f(u)$ is a strictly convex function of $u$ :

$$
f^{\prime \prime}(u) \geq a>0 \text {. }
$$

A planar viscous shock wave is a solution of (1) of the form $u(x, y, t)=$ $\phi(x-s t)$. This $\phi$ satisfies (see e.g. [Ge])

$$
\begin{gathered}
-s \phi^{\prime}+f(\phi)^{\prime}=\phi^{\prime \prime}, \\
\phi(x) \rightarrow u_{l} \text { as } x \rightarrow-\infty, \quad \phi(x) \rightarrow u_{r} \quad \text { as } x \rightarrow \infty,
\end{gathered}
$$

Received by the editors August 17, 1987 and, in revised form, November 15, 1987. 1980 Mathematics Subject Classification (1985 Revision). Primary 35L65, 76N10. 
where $\left(u_{l}-u_{r}\right) s=f\left(u_{l}\right)-f\left(u_{r}\right)$. It is clear that (3) and (4) are inconsistent with (2) unless $u_{l}>u_{r}$ and $\phi^{\prime}(x)<0$ for all $x$.

Theorem. If $u_{l}-u_{r}$ is small enough, for any $p>1$ there is a $c>0$ so that if

$$
\sum_{|\alpha| \leq 2} \cdot \iint\left(1+x^{2}\right)^{p}\left[\partial^{\alpha}(u(x, y, 0)-\phi(x))\right]^{2} d x d y \leq c,
$$

then

$$
\iint(u(x, y, t)-\phi(x-s t))^{2} d x d y \rightarrow 0 \text { as } t \rightarrow \infty .
$$

We begin by outlining the main energy estimate. The remaining details of the proof mostly could be supplied by a reader familiar with [KM, Go], or [Li]. The strategy is suggested by Liu's proof [Li] for systems in one dimension. To exploit the condition $(d / d x) f^{\prime}(\phi(x))<0$ we want to integrate in $x$ (see $\S 1$ of [Go]). It will be impossible to write the perturbation as $u-\phi=U_{x}$, with $U \in L_{2}$, unless we can arrange for something like $\int_{-\infty}^{\infty}(u(x, y, t)-\phi(x)) d x=0$. For this, we need a shift $\delta(y, t)$. Now,

$$
\int_{-\infty}^{\infty}(u(x, y, t)-\phi(x-\delta)) d x=\int_{-\infty}^{\infty}(u(x, y, t)-\phi(x)) d x+\delta \cdot\left(u_{l}-u_{r}\right),
$$

so it is possible to choose $\delta(y, t)$ so that

$$
\int_{-\infty}^{\infty}(u(x, y, t)-\phi(x-\delta(y, t))) d x=0 \text { for all } y, t
$$

The program, then, is to decompose the solution as

$$
u(x, y, t)=\phi(x-\delta(y, t))+U_{x}(x, y, t)
$$

and to seek $L_{2}$ estimates for $\delta$ and $U$.

The estimate is simpler if we set $g=0$ and $s=0$. If we insert (6) into (1) and use (3), we find

$$
\begin{aligned}
& -\phi^{\prime}(x-\delta(y, t)) \delta_{t}(y, t)+U_{t x}+\left(f^{\prime}(\phi) U_{x}+q(\phi, u) U_{x}^{2}\right)_{x} \\
& \quad=-\phi^{\prime}(x-\delta(y, t)) \delta_{y y}(y, t)+\phi^{\prime \prime}(x-\delta) \delta_{y}^{2}+U_{x x x}+U_{y y x} .
\end{aligned}
$$

Here we have used the Taylor expansion $f(\phi+v)=f(\phi)+f^{\prime}(\phi) v+q(\phi, v) v^{2}$. We will often leave out function arguments once they are clear from the context. The reason for this will quickly become clear to the reader who tries to put all the arguments in. Equation (7) will integrate in $x$ if the terms proportional to $\phi^{\prime}$ cancel. This leads to a system of equations that is equivalent to (1), namely

$$
\begin{gathered}
U_{t}+f^{\prime}(\phi) U_{x}+q(\phi, u) U_{x}^{2}=U_{x x}+U_{y y}+\phi^{\prime} \delta_{y}^{2}, \\
\delta_{t}=\delta_{y y} .
\end{gathered}
$$

For now, let us assume that

$$
\sup \left|q(\phi, u) U_{x}\right| \leq \frac{1}{2}, \quad \sup \left|\delta_{y}\right| \leq \frac{a}{2}, \quad \sup \left|\delta_{y}\right| \leq \frac{1}{u_{l}-u_{r}}
$$


where $a$ is from (2). Then multiplication and integration by parts leads to

(10) $\frac{d}{d t} \iint U^{2} d x d y \leq-\iint\left(U_{x}^{2}+U_{y}^{2}\right) d x d y-a \iint\left|\phi^{\prime}(x-\delta)\right| U^{2} d x d y$

$$
+2 \sup \left|\delta_{y}\right| \cdot \iint|U|\left|\delta_{y}\right|\left|\phi^{\prime}\right| d x d y
$$

$$
\frac{d}{d t} \int \delta^{2} d y \leq-2 \int \delta_{y}^{2} d y
$$

Considering the last term in (10) as an inner product in the weighted $L_{2}$ space with weight $\left|\phi^{\prime}(x-\delta)\right|$, the Cauchy-Schwarz inequality gives

$$
\iint|U|\left|\delta_{y}\right|\left|\phi^{\prime}\right| d x d y \leq \iint U^{2}\left|\phi^{\prime}\right| d x d y+\iint \delta_{y}^{2}\left|\phi^{\prime}\right| d x d y .
$$

If we integrate the $\delta_{y}^{2}$ term in $x$ using the bounds on $\left|\delta_{y}\right|$, then (9) and (10) combine to give

$$
\begin{aligned}
& \frac{d}{d t}\left(\iint U^{2} d x d y+\int \delta^{2} d y\right) \\
& \quad \leq-\iint\left(U_{x}^{2}+U_{y}^{2}\right) d x d y-\frac{a}{2} \iint\left|\phi^{\prime}(x-\delta)\right| U^{2} d x d y-\int \delta_{y}^{2} d y
\end{aligned}
$$

From experience with the one dimensional case we recognize that (12) will lead to a stability theorem, given enough patience.

\section{THE BASIC ENERGY ESTIMATE}

For the general case, $g \neq 0$, we need some preliminary normalizations. Each one is done by choosing new primed independent variables. After each normalization we will drop the primes and assume that the normalization holds.

Normalization 1. Set $s=0$. Choose

$$
t^{\prime}=t, \quad x^{\prime}=x-s t, \quad y^{\prime}=y .
$$

In the primed variables, $f\left(u_{l}\right)=f\left(u_{r}\right)$. For weak shocks this implies that $f^{\prime}(\phi(x))=O\left(u_{l}-u_{r}\right)$ for all $x$.

Normalization 2. Make $g^{\prime \prime}$ small. This works for sufficiently weak shocks (see the discussion at the end). The substitution

$$
t^{\prime}=t, \quad x^{\prime}=x, \quad y^{\prime}=y+\rho x,
$$

with $\rho=-g^{\prime \prime}(\phi(0)) / f^{\prime \prime}(\phi(0))$, transforms (1) into (dropping the primes)

$$
u_{t}+f(u)_{x}+g(u)_{y}=u_{x x}+2 \rho u_{x y}+\left(1+\rho^{2}\right) u_{y y},
$$

where $g^{\prime \prime}(\phi(x))=O\left(u_{l}-r_{r}\right)$ for all $x$. There is a positive constant $b$ depending on $\rho$ so that

$$
\xi^{2}+2 \rho \xi \eta+\left(1+\rho^{2}\right) \eta^{2}=(\xi+\rho \eta)^{2}+\eta^{2} \geq b\left(\xi^{2}+\eta^{2}\right) .
$$


Normalization 3. Make $g(\phi(x)) \in L_{2}(R)$. Use

$$
t^{\prime}=t, \quad x^{\prime}=x, \quad y^{\prime}=y-\sigma t
$$

where $\sigma$, defined by $\sigma=g\left(u_{l}\right)-g\left(u_{r}\right)$, is the mean transverse wave speed. We also add a constant to $g$, which does not change (1), to get $g\left(u_{l}\right)=g\left(u_{r}\right)=0$. From this we have $g^{\prime}\left(\left(u_{l}-u_{r}\right) / 2\right)=O\left[\left(u_{l}-u_{2}\right)^{2}\right]$, so, in view of Normalization 2, $g^{\prime}(\phi(x))=O\left[\left(u_{l}-u_{r}\right)^{2}\right]$ for all $x$ and

$$
|g(\phi(x))| \leq c\left(u_{l}-u_{r}\right)^{2} \cdot \begin{cases}\left(u_{l}-\phi(x)\right) & \text { if } x \leq 0 \\ \left(\phi(x)-u_{r}\right) & \text { if } x \geq 0\end{cases}
$$

But (2) implies that

$$
\left|u_{l}-\phi(x)\right| \leq \frac{c}{u_{l}-u_{r}}\left|\phi^{\prime}(x)\right| \text { if } x<0
$$

and therefore, for weak shocks, that

$$
|g(\phi(x))| \leq c\left(u_{l}-u_{r}\right) \cdot\left|\phi^{\prime}(x)\right| \text { for all } x .
$$

Let us calculate the analogue of (12) when $g \neq 0$ :

$$
\begin{aligned}
-\phi^{\prime} \delta_{t}+ & U_{t x}+\left(f^{\prime}(\phi) U_{x}\right)_{x}+\left(q U_{x}^{2}\right)_{x}-g(\phi)_{x} \delta_{y} \\
& +\left(g^{\prime}(\phi) U\right)_{y x}-\left(g^{\prime \prime}(\phi) \phi^{\prime} U\right)_{y}+\left(r U_{x}^{2}\right)_{y} \\
= & U_{x x x}+2 \rho U_{x y x}+\left(1+\rho^{2}\right) U_{y y x}-2 \rho \phi^{\prime \prime} \delta_{y} \\
& -\left(1+\rho^{2}\right) \phi^{\prime} \delta_{y y}+\left(1+\rho^{2}\right) \phi^{\prime \prime} \delta_{y}^{2} .
\end{aligned}
$$

Here $q=q(\phi, u)$ and $r=r(\phi, u)$ are bounded if $u$ is small enough. We assume that

$$
|q(\phi, u)| \leq d, \quad|r(\phi, u)| \leq d \text { for all } x, y, t .
$$

To integrate (15) in $x$ we need the decompositions

$$
\text { (16) }-g^{\prime \prime}(\phi(x-\delta(y, t))) \cdot \phi^{\prime}(x-\delta) U(x, y, t)=\alpha(y, t) \phi^{\prime}(x-\delta)+V_{x}(x, y, t)
$$

and

$$
r U_{x}^{2}=\beta(y, t) \phi^{\prime}(x-\delta)+W_{x}
$$

with $V$ and $W$ in $L_{2}$. Then instead of (8) and (9) we have

$$
\begin{aligned}
U_{t}+ & f^{\prime}(\phi) U_{x}+q U_{x}^{2}-g(\phi) \delta_{y}-\alpha \phi^{\prime} \delta_{y}+\left(g^{\prime}(\phi) U\right)_{y}+V_{y}+\beta \phi^{\prime} \delta_{y}+W_{y} \\
& =U_{x x}+2 \rho U_{x y}+\left(1+\rho^{2}\right) U_{y y}-2 \rho \phi^{\prime} \delta_{y}+\left(1+\rho^{2}\right) \phi^{\prime} \delta_{y}^{2},
\end{aligned}
$$

and

$$
\delta_{t}-\alpha_{y}-\beta_{y}=\left(1+\rho^{2}\right) \delta_{y y}
$$


We must examine the decompositions (16) and (17) in more detail. We will use the convention $\psi(x-\delta)=-\phi^{\prime}(x-\delta)$ so that $\psi$ is a positive weight function with $\int_{-\infty}^{\infty} \psi(x) d x=u_{l}-u_{r}$. In (16) we have

$$
\alpha(y, t)=\int_{-\infty}^{\infty} \psi(x-\delta(y, t)) \bar{U}(x, y, t) d x / \int \psi,
$$

where $\bar{U}=g^{\prime \prime}(\phi) U$, so Cauchy-Schwarz gives the bound for $\alpha$ :

(20)

$$
\alpha^{2}=\left(\int \psi \bar{U}\right)^{2} /\left(\int \psi\right)^{2} \leq \int 1^{2} \psi \cdot \int \bar{U}^{2} \psi /\left(\int \psi\right)^{2} \leq \int \bar{U}^{2} \psi / \int \psi .
$$

Using this we have

$$
\int(\bar{U}+\alpha)^{2} \psi \leq 2 \int \bar{U}^{2} \psi+2 \alpha^{2} \int \psi \leq 4 \int \bar{U}^{2} \psi .
$$

Temporarily forgetting the $y$ dependence, the problem of bounding $\alpha$ and $V(x)$ can be restated as follows. We have $l^{\prime}(x)=m(x) \psi(x)$ with

$$
\int m(x) \psi(x) d x=0
$$

and we want the bound $\int l^{2} \leq$ const $\cdot \int m^{2} \psi$. But $m^{2} \psi=l^{\prime 2} / \psi$ so the desired estimate follows from the following lemma. The lemma does not use the condition $\int m \psi=0$ and it applies only for $x \leq 0$. The same lemma holds for $x \in[0, \infty)$ if we integrate from $\infty$ instead of $-\infty$. The two indefinite integrals will agree and satisfy an estimate on $(-\infty, \infty)$ if $\int m \psi=0$. Applying the lemma to each $y$ separately we get

$$
\iint V^{2} \leq A_{2} \iint U^{2} \psi
$$

with

$$
A_{2}=4 \sup _{x}\left|g^{\prime \prime}(\phi(x))\right| \cdot \int_{-\infty}^{\infty}|x||\psi(x)| d x .
$$

Lemma 1. Assume that $l(x) \in H_{1}(-\infty, 0)$ (the Sobolev space). Then

$$
\int_{-\infty}^{0} l^{2}(x) \leq A_{1} \cdot \int_{-\infty}^{0} l^{\prime}(x)^{2} / \psi(x) d x
$$

where $A_{1}=\int_{-\infty}^{0}|x| \psi(x) d x$.

Proof. Since $l(x)=\int_{-\infty}^{x} l^{\prime}(\xi) d \xi$ we have

$$
l^{2}(x)=\left(\int_{-\infty}^{x} \frac{l^{\prime}(\xi)^{2}}{\sqrt{\psi(\xi)}} \cdot \sqrt{\psi(\xi)} d \xi\right)^{2} \leq \int_{-\infty}^{0} \frac{l^{\prime}(\xi)^{2}}{\psi(\xi)} d \xi \cdot \int_{-\infty}^{x} \psi(\xi) d \xi
$$

The lemma follows on integrating in $x$. 
Our bound for $W$ is similar but uses the Hilbert-Schmidt norm [DS]. An integral kernel $K(x, \xi)$ is in the Hilbert-Schmidt class if

$$
|K|_{\mathrm{HS}}^{2}=\iint K^{2}(x, \xi) d x d \xi<\infty .
$$

In that case the corresponding operator $K: l \rightarrow m$, given by

$$
m(x)=\int K(x, \xi) l(\xi) d \xi,
$$

is bounded on $L_{2}$. This is because

$$
|K|_{L_{2}}^{2}=\sup \frac{(m, K l)^{2}}{|l|_{L_{2}}^{2} \cdot|m|_{L_{2}}^{2}}
$$

and, using Cauchy-Schwarz on the $(x, \xi)$ integral,

$$
\begin{aligned}
(m, K l)^{2} & =\left(\iint m(x) K(x, \xi) l(\xi) d x d \xi\right)^{2} \\
& \leq \iint m^{2}(x) l^{2}(\xi) d x d \xi \cdot \iint K^{2}(x, \xi) d x d \xi \leq|m|_{L_{2}}^{2} \cdot|l|_{L_{2}}^{2} \cdot|K|_{\mathrm{HS}}^{2},
\end{aligned}
$$

so the norm of $K$ as an operator on $L_{2}$ has the bound $|K|_{L_{2}} \leq|K|_{\mathrm{HS}}$.

Now, just as for $\alpha$ we have

$$
\beta^{2} \leq\left(\int r U_{x}^{2}\right)^{2} /\left(\int \psi\right)^{2}
$$

so $\int\left(r U_{x}-\beta \psi\right)^{2} \leq 4 \int r U_{x}^{2}$.

The counterpart of Lemma 1 is

Lemma 2. Assume that $l \in L_{2}(-\infty, 0)$ and that $l^{\prime}=r m^{\prime 2}$ with $|r| \leq d$. Then

$$
\int_{-\infty}^{0} l^{2}(x) d x \leq A_{3} \int_{-\infty}^{0} m^{\prime}(x)^{2} d x
$$

where $A_{3}=d^{2} \int_{-\infty}^{0}|x| m^{\prime}(x)^{2} d x$.

Proof. We have

$$
l(x)=\int_{-\infty}^{x} r m^{\prime}(\xi)^{2} d \xi=\int_{-\infty}^{0} K(x, \xi) m^{\prime}(\xi) d \xi,
$$

where

$$
K(x, \xi)= \begin{cases}0 & \text { if } \xi>x \\ r m^{\prime}(\xi) & \text { if } \xi \leq x\end{cases}
$$

Then

$$
\begin{aligned}
|K|_{\mathrm{HS}}^{2} & =\int_{-\infty}^{0} \int_{-\infty}^{0} K^{2}(x, \xi) d x d \xi \leq \sup |r|^{2} \int_{-\infty}^{0} m^{\prime}(\xi)^{2} \int_{\xi}^{0} d x d \xi \\
& \leq d^{2} \int_{-\infty}^{0}|\xi| m^{\prime}(\xi)^{2} d \xi
\end{aligned}
$$

which proves the lemma. 
Several terms arise when we multiply (18) by $U$ and integrate over $x$ and $y$. First we have

$$
\iint f^{\prime}(\phi) U U_{x}=-\frac{1}{2} \iint f^{\prime \prime}(\phi) \phi^{\prime} U^{2} \geq \frac{a}{2} \iint U^{2} \psi .
$$

Next, using (23),

$$
\left|\iint q U U_{x}^{2}\right| \leq \frac{b}{10} \quad \text { if } \sup |U| \leq \frac{b}{10 d}
$$

Observe (using (2)) that there is a $c>0$ so that if $x \leq 0$ then $\psi(x)>$ $c\left|\phi(x)-u_{l}\right|$. Now using Cauchy-Schwarz as above,

$$
\left|\iint U g^{\prime}(\phi) \psi \delta_{y}\right| \leq \frac{a}{20} \iint U^{2} \psi+\frac{1}{10} \int \delta_{y}^{2} .
$$

Here and often below we use the hypothesis that $u_{l}-u_{r}$ is sufficiently small without explicitly stating it. Similarly,

$$
\left|\iint U \alpha \psi \delta_{y}\right| \leq \frac{a}{20} \iint U^{2} \psi+\frac{1}{10} \int \delta_{y}^{2} \text { if } \sup |\alpha| \leq \frac{a}{10} .
$$

From (21) and (22) we see that

$$
\left|\iint U V_{y}\right| \leq \frac{a}{10} \iint U^{2} \psi+\frac{b}{10} \iint U_{y}^{2} U_{y}^{2} \text { if } A_{2} \leq \frac{a}{50 \cdot b} .
$$

Continuing:

$$
\begin{aligned}
& \left|\iint U \beta \psi \delta_{y}\right| \leq \frac{\sup |U|}{2}\left(\int \psi \cdot \iint \beta^{2} \psi+\frac{1}{\int \psi} \iint \delta_{y}^{2} \psi\right), \\
& \left|\iint U \beta \psi \delta_{y}\right| \leq \frac{b}{10} \iint U_{x}^{2}+\frac{1}{10} \int \delta_{y}^{2} \text { if } \sup |U| \leq \frac{b}{5 d},
\end{aligned}
$$

and, from Lemma 2 ,

$$
\left|\iint U W_{y}\right|=\left|\iint U_{y} W\right| \leq \frac{b}{10} \iint U_{y}^{2}+\frac{b}{10} \iint U_{x}^{2} \text { if } A_{3} \leq \frac{b}{10} .
$$

Finally,

$$
\left|2 \rho \iint U \psi \delta_{y}\right| \leq 10 \rho \int \psi \cdot \iint U^{2} \psi+\frac{1}{10} \int \delta_{y}^{2}
$$

and

$$
\left|\iint U\left(1+\rho^{2}\right) \psi \delta_{y}^{2}\right| \leq \frac{a}{20} \iint U^{2} \psi+\frac{1}{10} \int \delta_{y}^{2} \quad \text { if } \sup \left|\delta_{y}\right| \leq 1 .
$$

For the $\delta$ equation (19) we have

$$
\left|\int \delta \alpha_{y}\right|=\left|\int \delta_{y} \alpha\right| \leq \frac{\sup \left|g^{\prime \prime}\right|}{u_{l}-u_{r}} \cdot \iint \delta_{y} \psi U \leq \frac{1}{10} \int \delta_{y}^{2}+\frac{a}{20} \iint \psi U^{2}
$$


and

$$
\left|\int \delta \beta_{y}\right|=\left|\int \delta_{y} \beta\right| \leq \frac{1}{10} \int \delta_{y}^{2}+\frac{b}{10} \iint U_{x}^{2}
$$

if

$$
\sup _{y}\left|\int U_{x}^{2}(x, y, t) d y\right| \leq \frac{b}{5 d}\left(u_{l}-r_{r}\right)^{2} .
$$

If we assemble (24)-(35) we get the basic energy estimate

$$
\frac{d}{d t}\left(\iint U^{2}+\int \delta^{2}\right) \leq-b \iint\left(U_{x}^{2}+U_{y}^{2}\right)-\frac{a}{2} \iint U^{2} \psi-\int \delta_{y}^{2} .
$$

The energy estimates above and below assume that $\delta(y, 0) \in H_{2}(R)$ and that $U(x, y, 0) \in L_{2}\left(R^{2}\right)$. These are consequences of the hypotheses of the theorem. For $\delta$, note that, from (5),

$$
\delta(y, 0)=\frac{1}{u_{l}-u_{r}} \int_{-\infty}^{\infty}(u(x, y, 0)-\phi(x)) d x,
$$

so that, using the Schwarz inequality,

$$
\begin{aligned}
\left|\delta_{y y}\right|^{2} & \leq c \cdot\left(\int_{-\infty}^{\infty}\left|\partial_{y}^{2}(u(x, y, 0)-\phi(x))\right| d x\right)^{2} \\
& \leq c \cdot \int_{-\infty}^{\infty}\left(1+x^{2}\right)\left|\partial_{y}^{2}(u(x, y, 0)-\phi(x))\right|^{2} d x \cdot \int_{-\infty}^{\infty} \frac{1}{1+x^{2}} d x .
\end{aligned}
$$

The assertion about $U$ is the only use of $p>1$ in the statement of the theorem. We use the formalism of Lemma 2. Suppose that

$$
a(x)=\int_{x}^{\infty} b(\xi) d \xi=\int_{0}^{\infty} K(x, \xi) c(\xi) d \xi,
$$

where $c(x)=\left(1+x^{2}\right)^{p / 2} b(x)$, and

$$
K(x, \xi)= \begin{cases}\left(1+x^{2}\right)^{-p / 2} & \text { if } \xi>x, \\ 0 & \text { if } \xi \leq x .\end{cases}
$$

Then $a \in L_{2}[0, \infty)$, since $\int_{0}^{\infty} \int_{0}^{\infty} K^{2}(x, \xi) d x d \xi<\infty$ and $\int_{0}^{\infty} c^{2}(x) d x=$ $\int_{0}^{\infty}\left(1+x^{2}\right)^{p} b^{2}(x) d x<\infty$.

\section{Higher estimates}

The purpose of this section is to finish the proof by showing that when the basic estimate (37) holds, and if the initial perturbation is small enough, then the assumptions made in deriving (37) are true. This will then imply that (37) is true a little longer and hence forever. This is a form of continuous induction which we treat sketchily here since it is given fully in [Go] and in many other places. The various assumptions are consequences of energy estimates for higher derivatives of $U$ and $\delta$ and the Sobolev lemma [F]. We establish the higher 
estimates by the same kind of arguments that we used to establish (37) except that (37) itself gives one the space time integrals

$$
\int_{0}^{T} \iint\left(|\nabla U|^{2}(x, y, t)+U^{2} \psi\right) d x d y d t+\int_{0}^{T} \int \delta_{y}^{2}(y, t) d y d t
$$

so less care is needed.

We start with a bound involving $\bar{u}=U_{x}=u-\phi$. To bound $A_{3}$ in Lemma 2 and (31), we estimate

$$
\iint \bar{u}^{2}(x, y, t) w(x) d x d y
$$

where $w(x)=\sqrt{1+x^{2}}$. The precise form of $w$ is not important. It only matters that $w(x) \geq|x|$, that $w(x) \geq 1$, and that $\left|w^{\prime}(x)\right| \leq 1$ for all $x$. From (15) and (19) we have

$$
\begin{aligned}
\phi^{\prime} \alpha_{y} & +\phi^{\prime} \beta_{y}+\bar{u}_{t}+\left(f^{\prime}(\phi) \bar{u}\right)_{x}+\left(q \bar{u}^{2}\right)_{x}-g(\phi)_{x} \delta_{y}+\left(g^{\prime}(\phi) \bar{u}\right)_{y}+\left(r \bar{u}^{2}\right)_{y} \\
& =\bar{u}_{x x}+2 \rho \bar{u}_{x y}+\left(1+\rho^{2}\right) \bar{u}_{y y}-2 \rho \phi^{\prime \prime} \delta_{y}+\left(1+\rho^{2}\right) \phi^{\prime} \delta_{y}^{2} .
\end{aligned}
$$

We multiply by $w \bar{u}$, integrate by parts, and bound all the terms by $\iint \bar{u}^{2}, \int \delta_{y}^{2}$, $\iint U^{2} \psi$, and $\varepsilon \iint|\nabla \bar{u}|^{2} w$ to get the estimate

$$
\begin{aligned}
& \iint \bar{u}(x, y, T) w(x) d x d y \leq \iint \bar{u}(x, y, 0) w(x) d x d y \\
& \quad-\frac{b}{2} \int_{0}^{T} \iint|\nabla \bar{u}|^{2} w+c \cdot\left(\int_{0}^{T} \iint \bar{u}^{2}+\int_{0}^{T} \iint U^{2} \psi+\int_{0}^{T} \int \delta_{y}^{2}\right) .
\end{aligned}
$$

Some of the details are as follows:

$$
\begin{aligned}
\left|\iint w \bar{u} \psi \alpha_{y}\right| & \leq\left|\iint w \bar{u}_{y} \psi \alpha\right|+\left|\iint w \bar{u} \psi^{\prime} \delta_{y} \alpha\right| \\
& \leq \iint w \bar{u}_{y}^{2}+\iint w \psi^{2} \alpha^{2}+\sup _{y}\left|\delta_{y}\right| \cdot\left(\iint w \bar{u}^{2}+\iint w \psi^{\prime 2} \alpha^{2}\right) .
\end{aligned}
$$

But

$$
\iint w \psi^{2} \alpha^{2} \leq \sup _{y} \int_{-\infty}^{\infty} w(x) \psi^{2}(x-\delta) d x \cdot \int \alpha^{2}(y, t) d y .
$$

If $|\delta| \leq 1$ then

$$
\sup _{y} \int w(x) \psi^{2}(x-\delta) d x=O\left(u_{l}-u_{r}\right) .
$$

Also, $\int \alpha^{2} \leq c \iint U^{2} \psi$. The $\iint w \bar{u} \psi \beta_{y}$ term is treated in the same way. Now

$$
\iint w \bar{u}\left(f^{\prime}(\phi) \bar{u}\right)_{x}=\frac{1}{2} \iint\left(w \psi f^{\prime \prime}(\phi)-w_{x} f^{\prime}(\phi)\right) \bar{u}^{2} \leq c \iint \bar{u}^{2} .
$$

Next

$$
\iint w \bar{u}\left(q \bar{u}^{2}\right)_{x}=-\iint w_{x} \bar{u}^{3} q-\iint w q \bar{u}^{2} \bar{u}_{x} .
$$


We set $Q(\phi, \bar{u})=\int_{0}^{\bar{u}} q(\phi, v) v^{2} d v$ so that $Q_{\bar{u}}(\phi, \bar{u})=q \bar{u}^{2},\left|Q_{\phi}\right| \leq c|\bar{u}|^{3}$, and

$$
\begin{aligned}
\iint w q \bar{u}^{2} \bar{u}_{x} & =\iint w Q(\phi, \bar{u})_{x}-\iint w Q_{\phi} \psi \\
& =\iint w_{x} Q+w Q_{\phi} \psi \leq c \cdot \sup |\bar{u}| \cdot \iint \bar{u}^{2} .
\end{aligned}
$$

Continuing:

$$
\begin{gathered}
\iint w \bar{u} g_{x} \delta_{y} \leq \frac{1}{2} \iint \bar{u}^{2}+\frac{1}{2} \iint w^{2} \psi^{2} g^{\prime 2} \delta_{y}^{2} \leq \frac{1}{2} \iint \bar{u}^{2}+c \int \delta_{y}^{2}, \\
\iint w \bar{u}\left(g^{\prime}(\phi) \bar{u}\right)_{y}=-\frac{1}{2} \iint w \bar{u}^{2} g^{\prime \prime}(\phi) \psi \leq \iint \bar{u}^{2}+c \int \delta_{y}^{2},
\end{gathered}
$$

and

$$
\begin{aligned}
\iint w \bar{u}\left(r \bar{u}^{2}\right)_{y} & =-\iint w R_{\phi}(\phi, \bar{u}) \psi \delta_{y} \leq \iint \psi w R_{\phi}^{2}+\iint \psi w \delta_{y}^{2} \\
& \leq c\left(\sup \left|\bar{u}^{4}\right| \cdot \iint \bar{u}^{2}+\int \delta_{y}^{2}\right),
\end{aligned}
$$

where $R(\phi, \bar{u})=\int_{0}^{\bar{u}} r(\phi, v) v^{2} d v$. Treating the viscous terms as above we establish (39) for all $T$ for which $\sup |\bar{u}| \leq 1, \sup |\delta| \leq 1$, and $\sup \left|\delta_{y}\right| \leq 1$.

The next step is to bound $\int \delta_{y}^{2}$. As above, we multiply the $\delta_{y}$ equation

$$
\delta_{y t}+\alpha_{y y}+\beta_{y y}=\left(1+\rho^{2}\right) \delta_{y y y}
$$

by $\delta_{y}$ and bound the resulting terms by quantities, including $\iint|\nabla \bar{u}|^{2}$, that we now know to have finite space time integrals. First

$$
\int \delta_{y} \alpha_{y y}=-\int \delta_{y y} \alpha_{y} \leq \varepsilon \int \delta_{y y}^{2}+c(\varepsilon) \int \alpha_{y}^{2} .
$$

But $\alpha=\int g^{\prime \prime} \psi U / \int \psi$ so

$$
\alpha_{y}=\left\{-\delta_{y} \int g^{\prime \prime \prime}(\phi) \psi^{2} U-\delta_{y} \int g^{\prime \prime}(\phi) \psi^{\prime} U+\int g^{\prime \prime} \psi U_{y}\right\} / \int \psi
$$

Now

$\int g^{\prime \prime \prime} \psi^{2} U \leq c \cdot \sqrt{\int \psi^{2} \psi} \cdot \sqrt{\int U^{2} \psi}, \quad \int g^{\prime \prime} \psi^{\prime} U \leq c \cdot \sqrt{\int\left|\psi^{\prime}\right|} \cdot \sqrt{\int U^{2}\left|\psi^{\prime}\right|}$,

and

so

$$
\int g^{\prime \prime} \psi U_{y} \leq c \cdot \sqrt{\int \psi^{2}} \cdot \sqrt{\int U_{y}^{2}},
$$

$$
\alpha_{y}^{2} \leq c \cdot \sup \left|\delta_{y}\right| \cdot\left(\int \psi U^{2}+\int U_{y}^{2}\right) \text {. }
$$

Second,

$$
\beta_{y}=\frac{1}{\int \psi}\left\{-\delta_{y} \int r_{\phi} \psi \bar{u}^{2}+\int\left(r_{\bar{u}} \bar{u}+2 r\right) \overline{u u}_{y}\right\}
$$


The first term is bounded by

$$
c \cdot \sup \left|\delta_{y}\right| \cdot \sup |\bar{u}| \sqrt{\int \bar{u}^{2}}
$$

and the second by

$$
c \cdot \sqrt{\int \bar{u}^{2}(x, y, t) d x} \cdot \sqrt{\int \bar{u}_{y}^{2}(x, y, t) d x} .
$$

So, if $A_{3}, \bar{u}$, and $\delta_{y}$ are bounded, we get

$$
\int \beta_{y}^{2} \leq c \cdot \iint\left(\bar{u}^{2}+\bar{u}_{y}^{2}\right) .
$$

To control $\int \delta_{y y}^{2}$ (needed for $\left.\sup \left|\delta_{y}\right|\right)$ we use a bound for $\iint U_{y}^{2}$. This comes from differentiating (18) with respect to $y$, multiplying by $U_{y}$, and controlling the resulting terms as above. A few terms deserve special attention. First,

$$
\iint U_{y y} g(\phi) \delta_{y} \leq \varepsilon \iint U_{y y}^{2}+c(\varepsilon) \iint g(\phi)^{2} \delta_{y}^{2} \leq \varepsilon \iint U_{y y}^{2}+c \int \delta_{y}^{2} .
$$

From (16) we have

$$
-g^{\prime \prime \prime} \psi^{2} \delta_{y} U+g^{\prime \prime} \psi^{\prime} \delta_{y} U-g^{\prime \prime} \psi U_{y}+\alpha_{y} \psi-\alpha \psi^{\prime} \delta_{y}=V_{x y} .
$$

From the discussion around Lemma 1 we conclude that

$$
\int V_{y}^{2}(x, y, t) d x \leq c \cdot \int\left(U^{2}+U_{y}^{2}\right) \psi .
$$

Similarly, differentiating (17) and using Lemma 2 and the identity $U_{x}=\bar{u}$ leads to

$$
\int W_{y}^{2} \leq c \cdot \int\left(\bar{u}^{2}+\bar{u}_{y}^{2}\right) .
$$

Now, repeating the arguments in the previous paragraph, we get the desired bound for $\int \delta_{y y}^{2}$.

It remains to obtain bounds for derivatives of $\bar{u}$. If we differentiate (38) with respect to $y$, multiply by $w \bar{u}_{y}$, and integrate, most of the terms are handled as above except

$$
\begin{aligned}
\left.\mid \iint w \bar{u}_{y}\left(\psi \alpha_{y}\right)_{y}\right) \mid & =\left|\iint w \bar{u}_{y y} \psi \alpha_{y}\right| \leq \varepsilon \iint w \bar{u}_{y y}^{2}+c(\varepsilon) \iint w \psi^{2} \alpha_{y}^{2} \\
& \leq \varepsilon \iint w \bar{u}_{y y}^{2}+c \int \alpha_{y}^{2} .
\end{aligned}
$$

Now differentiating with respect to $x$ we get bounds for

$$
\sup _{t \leq T} \iint w(x)|\nabla \bar{u}(x, y, t)|^{2} d x d y,
$$

and

$$
\int_{0}^{T} \iint w(x)\left|D^{2} \bar{u}(x, y, t)\right|^{2} d x d y d t .
$$


Continuing in this way leads to a bound on $\sup _{t \leq T} \iint w(x)\left|D^{2} \bar{u}\right|^{2}$ which bounds $A_{2}$ in Lemma 2 as well as $\sup _{t \leq T}|\bar{u}|$ and the other quantities used in deriving (37).

\section{Discussion}

A low frequency linearized stability analysis suggests that the requirement of weak shocks is not necessary. Linearizing (1) about $u(x, y, t)=\phi(x)$ gives the linear equation

$$
v_{t}+\left(f^{\prime}(\phi) v\right)_{x}+\left(g^{\prime}(\phi) v\right)_{y}=v_{x x}+v_{y y} .
$$

We look for a separation of variables solution

$$
v(x, y, t)=e^{\tau t} e^{i \eta y} \hat{v}(x),
$$

where $\hat{v}$ satisfies

$$
\tau \hat{v}+\left(f^{\prime}(\phi) \hat{v}\right)_{x}+i \eta g^{\prime}(\phi) \hat{v}=\hat{v}_{x x}-\eta^{2} \hat{v} .
$$

For $\eta=0$ a solution with $\tau=0$ is given by $\hat{v}=\phi^{\prime}$. We therefore seek a small $\eta$ expansion

$$
\tau \sim \eta \tau_{1}-\eta^{2} \tau_{2}+\cdots, \quad \hat{v} \sim \phi^{\prime}+i \eta \hat{v}_{1}+\eta^{2} \hat{v}_{2}+\cdots .
$$

Substituting into (40) and equating powers of $\eta$ we find, to leading order,

$$
-i \tau_{1} \phi^{\prime}+\left(f^{\prime}(\phi)^{\prime} \hat{v}_{1}\right)_{x}+g(\phi)_{x}=\hat{v}_{1 x x} .
$$

Using Normalization 3 gives a zero constant of intergration in

$$
\hat{v}_{1 x}-f^{\prime}(\phi) \hat{v}_{1}=g(\phi(x)) \text {. }
$$

if $\tau_{1}=0$. We find $\tau_{2}$ from the $O\left(\eta^{2}\right)$ equation:

$$
\left(\tau_{2}+1\right) \phi^{\prime}=\hat{v}_{2 x x}-\left(f^{\prime}(\phi) \hat{v}_{2}\right)_{x}+g^{\prime}(\phi) \hat{v}_{1} .
$$

The integrability condition for this equation is

$$
-\tau_{2}+1=\frac{\int_{-\infty}^{\infty} g^{\prime}(\phi(x)) \hat{v}_{1}(x) d x}{\int_{-\infty}^{\infty} \phi^{\prime}(x) d x} .
$$

Since $\phi^{\prime}<0$, we will have $\tau_{2}>1$ when we show that

$$
\int g^{\prime}(\phi(x)) \hat{v}_{1}(x) d x>0 .
$$

But

$$
\int g^{\prime}(\phi) \hat{v}_{1}=\int g^{\prime}(\phi) \phi^{\prime} \frac{\hat{v}_{1}}{\phi^{\prime}}=\int g(\phi)_{x} \frac{\hat{v}_{1}}{\phi^{\prime}}=-\int \frac{g(\phi)}{\phi^{\prime}}\left(\hat{v}_{1 x}-\frac{\phi^{\prime \prime} \hat{v}_{1}}{\phi^{\prime}}\right) .
$$

Now, $\phi^{\prime \prime}=f(\phi)_{x}=f^{\prime}(\phi) \phi_{x}$, so

$$
\int g^{\prime}(\phi) \hat{v}_{1}=\int \frac{g(\phi)}{\phi^{\prime}}\left(\hat{v}_{1 x}-f^{\prime}(\phi) \hat{v}_{1}\right)=\int_{-\infty}^{\infty} \frac{g(\phi(x))^{2}}{-\phi^{\prime}(x)} d x>0,
$$

as claimed. 
A formal calculation based on the ansats and scaling

$$
u(x, y, t) \sim \phi\left(x-\delta\left(\varepsilon y, \varepsilon^{2} t\right)\right)+\varepsilon u_{1}+\varepsilon^{2} u_{2}+\cdots
$$

also leads to $\delta_{t}=\tau_{2} \delta_{y y}$, which we expect to describe the long time behavior of $\delta$.

Acknowledgement. This work grew out of a conversation with Victor Roytburd. Comments by a referee led to considerable improvement of the paper. The work was supported by an NSF presidential young investigator award with matching funds from AT\&T Bell Laboratories and Sun Microsystems, and by a Sloan foundation fellowship.

\section{REFERENCES}

[DS] N. Dunford and J. T. Schwartz, Linear operators, Wiley, New York, 1958.

[F] G. Folland, Introduction to partial differential equations, Princeton Univ. Press, Princeton, N.J., 1976.

[Ge] I. M. Gelfand, Some problems in the theory of quasilinear equations, Uspekhi Mat. Nauk (N.S.) 14 (1959), 87-158; English transl., Amer. Math. Soc. Transl. (2) 29 (1963).

[Go] J. Goodman, Nonlinear asymptotic stability of viscous shock profiles for conservation laws, Arch. Rational Mech. Anal. 95 (1986), 325-344.

[IO] A. M. Il' in and O. A. Oleinik, Behavior or the solution of the Cauchy problem for certain quasilinear equations for unbounded increase of the time, Amer. Math. Soc. Transl. (2) 42 (1964), 19-23.

[KM] S. Kawashima and A. Matsumura, Asymptotic stability of travelling wave solutions of systems for one-dimensional gas motion, Comm. Math. Phys. 101 (1985), 97-127.

[Li] T. P. Liu, Nonlinear stability of shock waves for viscous conservation laws, Mem. Amer. Math. Soc., vol. 328, 1985.

[Lu] G. Ludford (Ed.), Reacting flows: Combustion and chemical reators, Proc. '85 AMS/SIAM Summer Seminar in Appl. Math., Cornell Univ., Amer. Math. Soc., Providence, R.I., 1986.

[S] D. Sattinger, On the stability of waves of nonlinear parabolic systems, Adv. in Math. 22 (1976), 312-355.

Courant Institute of Mathematical Sciences, 251 Mercer Street, New York, New YORK 10012 